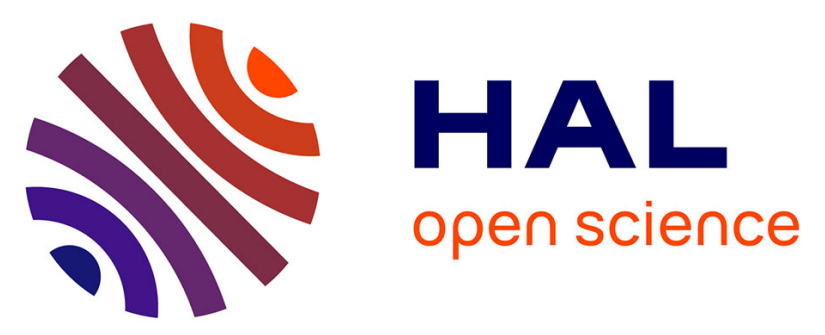

\title{
Hamiltonian four-field model for magnetic reconnection: nonlinear dynamics and extension to three dimensions with externally applied fields
}

Emanuele Tassi, Philip Morrison, Daniela Grasso, Francesco Pegoraro

\section{- To cite this version:}

Emanuele Tassi, Philip Morrison, Daniela Grasso, Francesco Pegoraro. Hamiltonian four-field model for magnetic reconnection: nonlinear dynamics and extension to three dimensions with externally applied fields. Nuclear Fusion, 2010, 50 (3), pp.034007. 10.1088/0029-5515/50/3/034007 . hal00477014

\author{
HAL Id: hal-00477014 \\ https://hal.science/hal-00477014
}

Submitted on 27 Apr 2010

HAL is a multi-disciplinary open access archive for the deposit and dissemination of scientific research documents, whether they are published or not. The documents may come from teaching and research institutions in France or abroad, or from public or private research centers.
L'archive ouverte pluridisciplinaire HAL, est destinée au dépôt et à la diffusion de documents scientifiques de niveau recherche, publiés ou non, émanant des établissements d'enseignement et de recherche français ou étrangers, des laboratoires publics ou privés. 


\title{
Hamiltonian four-field model for magnetic reconnection: nonlinear dynamics and extension to three dimensions with externally applied fields
}

\author{
E. Tassi ${ }^{1}$, P.J. Morrison ${ }^{2}$, D. Grasso ${ }^{3,4}$ and F. Pegoraro ${ }^{5}$ \\ ${ }^{1}$ Centre de Physique Théorique - UMR 6207, CNRS Luminy, Case 907, F - 13288, \\ Marseille Cedex 09, France \\ E-mail: tassi@cpt.univ-mrs.fr \\ 2 Department of Physics and Institute for Fusion Studies, University of Texas, \\ Austin, Texas 78712, United States \\ 3 CNR-INFM, Genova, Italy \\ ${ }^{4}$ Burning Plasma Reasearch Group, Dip. di Energetica, Politecnico di Torino, \\ CNISM, Italy \\ ${ }^{5}$ Dipartimento di Fisica E. Fermi and CNISM, Pisa, Italy
}

\begin{abstract}
.
The nonlinear dynamics of a two-dimensional model for collisionless magnetic reconnection is investigated both numerically and analytically. For very low values of the plasma $\beta$, parallel magnetic perturbations tend to be proportional to the vorticity perturbations, but as $\beta$ increases detachment of these quantities takes place. The subsequent difference between the structure of the vorticity and the parallel magnetic perturbations can be explained naturally in terms of the "normal" field variables that emerge from the noncanonical Hamiltonian theory of the model. A three-dimensional extension of the reconnection model is also presented, its Hamiltonian structure is derived, and the corresponding conservation properties are compared with those of the two-dimensional model. A general method for extending a large class of twodimensional fluid plasma models to three dimensions, while preserving the Hamiltonian structure, is then presented. Finally, it is shown how such models can also be extended, while preserving the Hamiltonian structure, to include externally applied fields, that can be used, for instance, for modelling resonant magnetic perturbations.
\end{abstract}




\section{Introduction}

Magnetic reconnection is believed to play a key role for many phenomena occurring in fusion plasmas, such as sawtooth oscillations in tokamaks and relaxation in reversed field pinches (see, e.g. $[1,2]$ ). In high temperature tokamak plasmas with low collision frequencies, electron inertia can provide an effective mechanism for breaking the frozenin condition of magnetohydrodynamics and thereby allow magnetic reconnection to take place. In order to understand the nonlinear dynamics of this kind of magnetic reconnection driven by electron inertia, a three-field fluid model for collisionless plasmas was obtained by Schep, Pegoraro and Kuvshinov (SPK) [3]. A two-dimensional (2D), two-field version of this model has been intensively investigated in Refs. $[4,5,6]$ and a 3D extension has been analyzed in Ref. [7]. Many of the obtained results have relied on knowledge of the noncanonical Hamiltonian structure (see, e.g. [8]) of the model equations $[3,9]$. Indeed, the Hamiltonian formulation led to the discovery of infinite families of Casimir invariants, associated with Lagrangian invariants. These invariants have made it possible to interpret and explain the formation of small scale structures in the plasma vorticity and current density [5].

A 2D, four-field model extension of the SPK model was obtained by Fitzpatrick and Porcelli (FP) in Ref. [10]. Unlike the SPK model, this model allows for magnetic and velocity perturbations in the direction of a guide field and also allows for finite $\beta$ regimes, where as usual $\beta$ is the ratio of the plasma and magnetic pressures. The Hamiltonian formulation of this model, derived in [11], proved to be useful for obtaining stability criteria [12], providing an unambiguous definition of negative energy modes [11], and interpreting numerical simulations [13, 14].

The present paper is a continuation of this line of research, it being devoted to the investigation of magnetic reconnection in collisionless plasmas, while taking advantage of the Hamiltonian formalism. The purpose of the paper is twofold: first, results from a numerical investigation of the nonlinear dynamics of the parallel magnetic perturbations, perturbations that are not taken into account in the SPK model but are present in the FP model, is described. Second, it is shown how to extend a large class of 2D fluid models (which includes the FP model) to 3D Hamiltonian models with the inclusion of externally applied fields. The motivation for the latter is to include, within the fluid Hamiltonian framework, for instance, the effect of resonant magnetic perturbations that can have an important impact on turbulent fluctuations and transport (see, e.g. [15, 16, 17]).

The paper is structured as follows. In Sec. 2 the FP model and its Hamiltonian structure are briefly recalled. Results of the numerical simulations are then presented and their interpretation in terms of Hamiltonian "normal fields" is given. In Sec. 3, the 3D extension of the FP model is presented and its Hamiltonian structure is discussed. Next, the conditions for extensions of $2 \mathrm{D}$ models to $3 \mathrm{D}$, with externally applied fields, are presented and a specific example is given. Finally, we conclude in Sec. 4. 


\section{2D four-field model}

\subsection{Four-field review}

In this section we first consider the cold-ion four-field model for magnetic reconnection in collisionless plasmas presented in [10]. This model is formulated in Cartesian coordinates $(x, y, z)$, it assumes the presence of a strong guide field of amplitude $B^{(0)}$ directed along the ignorable coordinate $z$, and a constant background electron pressure $P_{0}$. The model equations, in a dimensionless form, are

$$
\begin{aligned}
& \frac{\partial\left(\psi-d_{e}^{2} \nabla^{2} \psi\right)}{\partial t}+\left[\varphi, \psi-d_{e}^{2} \nabla^{2} \psi\right]-d_{\beta}[\psi, Z]=0, \\
& \frac{\partial Z}{\partial t}+[\varphi, Z]-c_{\beta}[v, \psi]-d_{\beta}\left[\nabla^{2} \psi, \psi\right]=0, \\
& \frac{\partial \nabla^{2} \varphi}{\partial t}+\left[\varphi, \nabla^{2} \varphi\right]+\left[\nabla^{2} \psi, \psi\right]=0, \\
& \frac{\partial v}{\partial t}+[\varphi, v]-c_{\beta}[Z, \psi]=0,
\end{aligned}
$$

where the bracket [,] is defined by $[f, g]=\hat{z} \cdot(\nabla f \times \nabla g)$. Equations (1)-(2) describe the evolution of the fields $\psi$ and $Z$, which determine the magnetic field through the expression $\mathbf{B}(x, y, t)=\nabla \psi \times \hat{z}+\left(B^{(0)}+c_{\beta} Z\right) \hat{z}$, where $c_{\beta}=\sqrt{\beta /(1+\beta)}$, with $\beta=(5 / 3) P_{0} / B^{(0)^{2}}$. The plasma velocity field $\mathbf{v}$ is determined by a stream function $\varphi$ and a parallel component $v$ by the expression $\mathbf{v}(x, y, t)=-\nabla \varphi \times \hat{z}+v \hat{\mathbf{z}}$. The time evolution of the velocity follows from Eqs. (3)-(4). The parameter $d_{e}$, appearing in (1), is the electron skin depth and is associated with the term responsible for breaking the frozen-in condition. The parameter $d_{\beta}$ is defined as $d_{\beta}=d_{i} c_{\beta}$, with $d_{i}$ corresponding to the ion skin depth.

Equations (1)-(4) can be derived starting from a two-fluid description of a plasma. More precisely, Eq. (1) originates from the electron momentum equation, Eq. (2) from the electron vorticity equation, whereas Eqs. (3) and (4) can be obtained from the plasma vorticity and parallel momentum equations, respectively.

The system (1)-(4) has a noncanonical Hamiltonian formulation [18, 11], the Hamiltonian functional being given by

$$
H=\frac{1}{2} \int_{\mathcal{D}} d^{2} x\left(d_{e}^{2} J^{2}+|\nabla \varphi|^{2}+v^{2}+|\nabla \psi|^{2}+Z^{2}\right)
$$

with $J=-\nabla^{2} \psi$, corresponding to the parallel current density, and $\mathcal{D}$ indicating the spatial domain of interest. The quantity (5) corresponds to the total energy of the system and it naturally possesses a kinetic energy part, corresponding to the first three terms in (5), and a magnetic energy part, given by the remaining two terms. The noncanonical Hamiltonian formulation of the FP model is completed by a Poisson bracket, whose rather lengthy expression, in terms of the variables $\psi-d_{e}^{2} \nabla^{2} \psi, \nabla^{2} \varphi, Z$ and $v$, can be found in [11]. We recall that this bracket has four infinite families of so-called Casimir invariants, which are defined as functionals $C$ of the field variables, that satisfy the 
relation $\{F, C\}=0$, where $\{$,$\} is the Poisson bracket and F$ is any functional of the field variables. Because they commute with any the Hamiltonian functional, Casimir invariants are constants of motion for the system. The families of Casimirs for the FP model are

$$
\begin{aligned}
C_{1} & =\int d^{2} x \mathcal{H}(D), \\
C_{2} & =\int d^{2} x \zeta \mathcal{F}(D), \\
C_{3} & =\int d^{2} x \tilde{g}_{+}\left(T_{+}\right), \\
C_{4} & =\int d^{2} x \tilde{g}_{-}\left(T_{-}\right),
\end{aligned}
$$

where $\mathcal{H}, \mathcal{F}, \tilde{g}_{+}$and $\tilde{g}_{-}$are arbitrary functions, and

$$
\begin{aligned}
& D=\psi_{e}+d_{i} v, \\
& \zeta=\nabla^{2} \varphi+\frac{d_{i}}{c_{\beta} d^{2}} Z, \\
& T_{+}=\frac{d_{i}^{2}}{2 c_{\beta} d^{3} d_{e}} \psi_{e}-\frac{d_{i} d_{e}}{2 c_{\beta} d^{3}} v-\frac{d_{i}}{2 c_{\beta} d^{2}} Z, \\
& T_{-}=-\frac{d_{i}^{2}}{2 c_{\beta} d^{3} d_{e}} \psi_{e}+\frac{d_{i} d_{e}}{2 c_{\beta} d^{3}} v-\frac{d_{i}}{2 c_{\beta} d^{2}} Z,
\end{aligned}
$$

where $\psi_{e}=\psi-d_{e}^{2} \nabla^{2} \psi$ and $d=\sqrt{d_{i}^{2}+d_{e}^{2}}$.

The form of the Casimirs (6)-(9) leads to an alternative set of field variables, that we call "normal" fields, according to the terminology introduced in [19], consisting of the variables $D, \zeta, T_{+}, T_{-}$, which are linear combinations of the original variables $\psi_{e}$, $\nabla^{2} \varphi, Z, v$. In terms of the normal fields, the Poisson bracket of the FP model takes the simple form

$$
\begin{array}{r}
\{F, G\}=\int d^{2} x\left(\zeta\left[F_{\zeta}, G_{\zeta}\right]+D\left(\left[F_{D}, G_{\zeta}\right]+\left[F_{\zeta}, G_{D}\right]\right)\right. \\
\left.+T_{-}\left[F_{T_{-}}, G_{T_{-}}\right]+T_{+}\left[F_{T_{+}}, G_{T_{+}}\right]\right),
\end{array}
$$

where subscripts indicate functional derivatives. The Hamiltonian (5) in terms of the normal fields becomes

$$
\begin{aligned}
H & =\int_{\mathcal{D}} d^{2} x\left[\frac{c_{\beta}^{2} d^{4}}{d_{i}^{2}}\left(T_{+}^{2}+T_{-}^{2}\right)+\frac{D^{2}}{2 d^{2}}-\frac{1}{2}\left(\zeta+T_{+}+T_{-}\right) \nabla^{-2}\left(\zeta+T_{+}+T_{-}\right)\right. \\
& \left.-\frac{1}{2}\left(\frac{d_{e}}{d^{2}} D+c_{\beta} d\left(T_{+}-T_{-}\right)\right) \mathcal{L}\left(\frac{d_{e}}{d^{2}} D+c_{\beta} d\left(T_{+}-T_{-}\right)\right)\right],
\end{aligned}
$$

where $\mathcal{L}$ is an operator defined by the relation $\mathcal{L} \psi_{e}=\psi$. The normal fields are convenient variables that make the conservation laws of the system more evident. Indeed, by making use of the normal fields, the FP model can be written as

$$
\frac{\partial D}{\partial t}=-[\varphi, D],
$$




$$
\begin{aligned}
& \frac{\partial \zeta}{\partial t}=-[\varphi, \zeta]+d^{-2}[D, \psi], \\
& \frac{\partial T_{ \pm}}{\partial t}=-\left[\varphi_{ \pm}, T_{ \pm}\right],
\end{aligned}
$$

where we have defined

$$
\varphi_{ \pm}:=\varphi \pm \frac{c_{\beta} d}{d_{e}} \psi .
$$

From the compact form of Eqs. (16)-(18), it possible to see that $D$ and $T_{ \pm}$are Lagrangian invariants advected by velocity fields determined by the stream functions $\varphi$ and $\varphi_{ \pm}$, respectively. The evolution equation for $\zeta$, on the other hand, possesses a source term that vanishes when $D$ is a flux function or when $d \rightarrow \infty$.

\subsection{Simulation results and interpretation}

The noncanonical Hamiltonian formulation and the knowledge of the Casimir invariants proved to be useful for investigating the nonlinear dynamics of the SPK reconnection model. From the knowledge of the Casimirs it was possible to deduce the conservation of the topology of field lines of the two Lagrangian invariants $G_{ \pm}[4]$ and to explain the formation of small scale structures in the plasma vorticity [5]. The two-field version of the SPK model investigated in $[4,5]$, can be obtained from the FP model by taking the limit $c_{\beta} \rightarrow 0$ and $d_{\beta} \rightarrow \rho_{s}$, where $\rho_{s}$ is the Larmor radius of ions with electron temperature. In this limit, Eq. (4) decouples from the system, whereas $Z=-\rho_{s} \nabla^{2} \varphi$ can be taken as the solution for $Z$, provided its compatibility with the initial conditions. From the expression $B_{z}=\left(B^{(0)}+c_{\beta} Z\right)$, one sees that the limit $c_{\beta} \rightarrow 0$, corresponds to suppressing parallel magnetic perturbations. The evolution of such perturbations for the FP model has not yet been investigated, in particular, with regard to its interpretation in terms of normal fields. In the following, we investigate the field $Z$ and its dependence on the value of $c_{\beta}$, in order to see how the nonlinear evolution of $Z$ develops in regimes with non-negligible values of $c_{\beta}$. Such a regime is not accessible in the SPK two-field model. Given that the FP model assumes $p=-c_{\beta} Z$, where $p$ is an electron pressure perturbation, the evolution of $Z$ can also provide information about the pressure evolution.

Equations (1)-(4) were solved numerically using a code based on a finite volume scheme. The equations are solved on a rectangular domain $\{(x, y):-2 \pi \leq x \leq 2 \pi,-\pi \leq$ $y \leq \pi\}$, subdivided into $1024 \times 1024$ grid points, with periodic boundary conditions imposed for both the $x$ and $y$ directions. We chose an initial equilibrium of the form $\psi_{0}(x)=1 / \cosh ^{2}(x), \varphi_{0}=0, Z_{0}=0, v_{0}=0$, which is unstable to reconnecting perturbations. We are mainly interested in seeing how the evolution is modified, in comparison to the SPK model, when one increases $c_{\beta}$ to non-negligible values. Two cases are considered: the first case has a very low value of $\beta$, with the parameter $d_{\beta}$ playing essentially the role of $\rho_{s}$ of the SPK model, while the second case has higher $\beta$. In both cases the value of the normalized electron skin depth is set equal to 0.24 . Note 

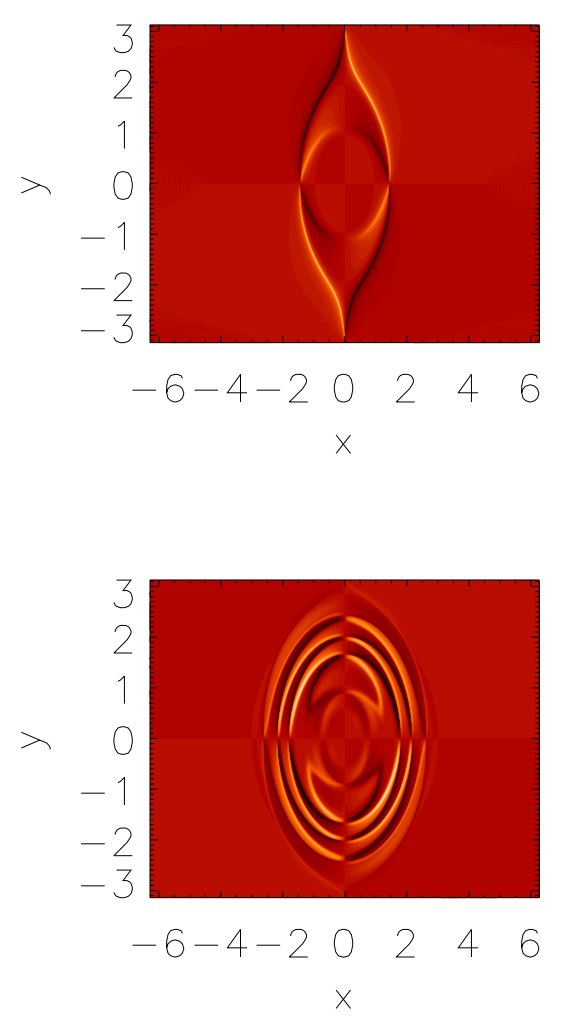
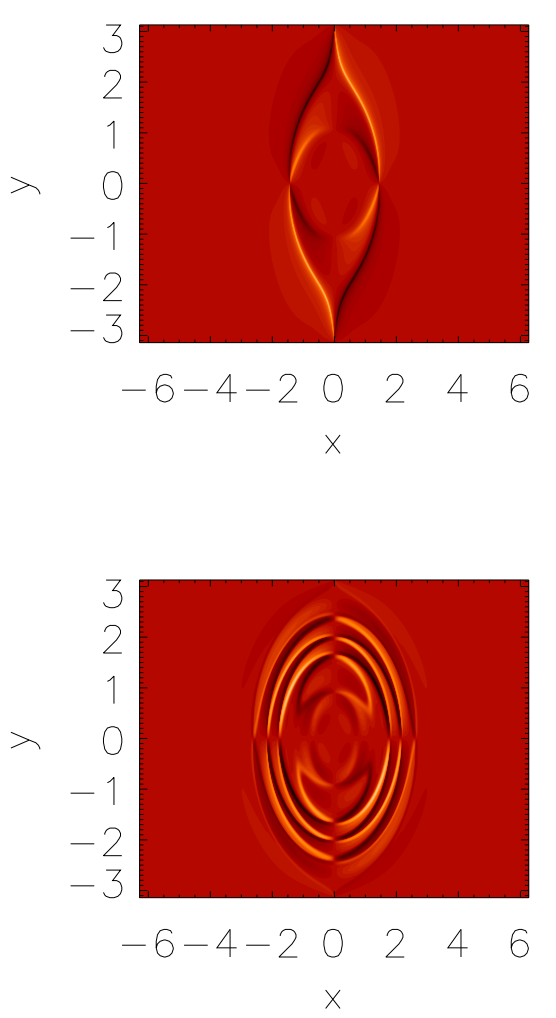

Figure 1. Contour plots of $\nabla^{2} \varphi$ (left column) and $Z$ (right column). The top row refers to $t=50$ Alfvèn times and the bottom row to $t=70$ Alfvèn times. Values of the parameters are $c_{\beta}=0.01, d_{\beta}=0.24$, and $d_{e}=0.24$. From the plots one can see that, because $Z_{0}=\nabla^{2} \varphi_{0}=0$, in this very low $\beta$ regime, parallel magnetic perturbations closely track the vorticity perturbations.

that, due to limitations in the numerical resolution, the electron/ion mass ratios for the two cases are slightly different.

In Fig. 1 contour plots of $\nabla^{2} \varphi$ and $Z$, for $c_{\beta}=0.01$, are compared. From the plots, it is evident that for these values the two fields are nearly identical. This is a direct consequence of considering a very low $\beta$ with finite $d_{\beta} \approx \rho_{s}$. As anticipated above, this suppresses the term $c_{\beta}[v, \psi]$ in Eq. (2), which couples to the parallel dynamics, and allows for the solution $Z=-d_{\beta} \nabla^{2} \varphi$. This requires the initial conditions for $Z$ and $\nabla^{2} \varphi$ to be identical, which is the case for the simulations under consideration. It is interesting to notice that the detachment of $Z$ from $-d_{\beta} \nabla^{2} \varphi$ is measured by the amplitude of the normal field $\zeta$. Indeed, for practical purposes, $d \approx d_{i}$, and therefore $\zeta \approx \nabla^{2} \varphi+Z / d_{\beta}$. In this regime, in which $d_{i}$ is large, the source term in Eq. (17) becomes negligible and consequently, because $\zeta(x, y, 0)=0$, the value of $\zeta$ remains equal to zero at all times. In other words, the difference between $Z$ and $-d_{\beta} \nabla^{2} \varphi$ cannot grow. Notice also that, if $\zeta=0$, then

$$
Z=-\frac{c_{\beta} d^{2}}{d_{i}}\left(T_{+}+T_{-}\right), \quad \nabla^{2} \varphi=T_{+}+T_{-}
$$



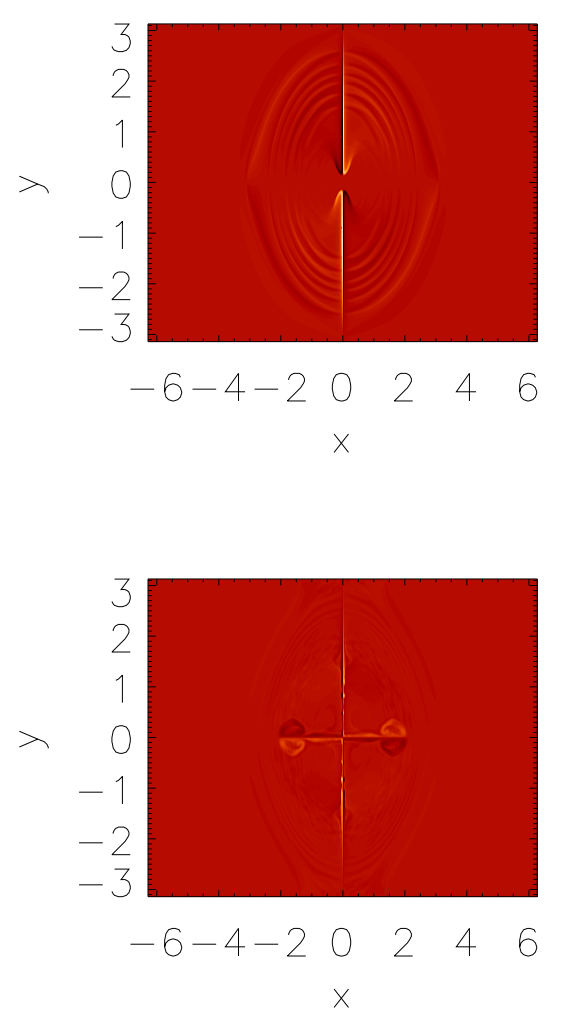
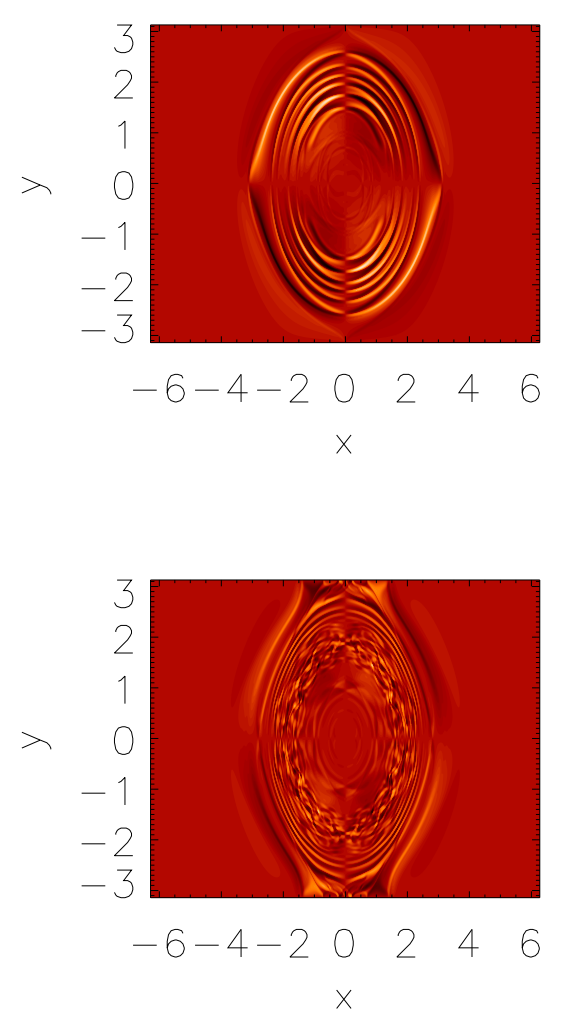

Figure 2. Contour plots of $\nabla^{2} \varphi$ (left column) and $Z$ (right column). The top row refers to $t=40$ Alfvèn times and the bottom row to $t=55$ Alfvèn times. Values of the parameters are $c_{\beta}=0.3, d_{\beta}=0.72$, and $d_{e}=0.24$. In this higher $\beta$ regime, the effect of the terms proportional to $c_{\beta}$ is no longer negligible and $Z$ detaches from $-d_{\beta} \nabla^{2} \varphi$.

Such a representation in terms of the Lagrangian invariants $T_{ \pm}$makes it possible to explain the formation of small scales in the vorticity and parallel magnetic field. As already explained in $[12,14]$ (and earlier in [5] for the two-field model) small scales are formed because of the stretching and filamentation of $T_{+}$and $T_{-}$, advected by velocity fields that rotate in opposite directions.

Figure 2, on the other hand, shows contour plots of $\nabla^{2} \varphi$ and $Z$ for a higher value of $\beta$. In this case it is possible to see that the parallel magnetic field (or, equivalently, the pressure) and the vorticity follow distinct evolutions. Indeed, $Z$ still undergoes a filamentation process, again corresponding to the stretching of the normal fields $T_{+}$and $T_{-}$, whose structures are now even less regular than in the very low $\beta$ regime. The vorticity, on the other hand, on top of the filamented structures, forms two vertical vortex sheets that collide and create vortices propagating in opposite directions along the $y=0$ line. The superposition of these two types of dynamics is naturally explained in terms of the normal fields. Indeed, the relations

$$
Z=-\frac{c_{\beta} d^{2}}{d_{i}}\left(T_{+}+T_{-}\right), \quad \nabla^{2} \varphi=\zeta+T_{+}+T_{-},
$$


make it possible to see that it is the growth of the field $\zeta$, that prevents $Z$ from being proportional to the vorticity, in this $\beta$ regime. The field $\zeta$, in particular, is the one responsible for the formation of vortex sheets, that can eventually become unstable $[20,12,14]$. The pressure and the parallel magnetic field, on the other hand, do not receive contributions from $\zeta$ and therefore they do not form such localized structures. Instead, they continue to transferring energy to smaller and smaller scales, in a manner similar to what happens in a phase mixing process.

\section{3D four-field model}

\subsection{Extension of the four-field model}

We assume now that the field variables $\psi, \varphi, Z$ and $v$ also depend on the spatial coordinate $z$. We introduce a smallness parameter $\epsilon$ defined as the ratio between the amplitudes of the poloidal magnetic field and of the strong guide magnetic field. We assume then the ordering

$$
\begin{aligned}
& B^{(0)}=\mathcal{O}\left(\frac{1}{\epsilon}\right) \gg 1, \\
& \psi \sim \varphi \sim Z \sim v \sim \frac{\partial}{\partial x} \sim \frac{\partial}{\partial y}=\mathcal{O}(1), \\
& \frac{\partial}{\partial z}=\mathcal{O}(\epsilon) \ll 1,
\end{aligned}
$$

which is consistent with the ordering of the FP model. If one then carries out, with these assumptions, the derivation of the FP model as described in Ref. [10], the resulting 3D model equations are:

$$
\begin{aligned}
& \frac{\partial\left(\psi-d_{e}^{2} \nabla_{\perp}^{2} \psi\right)}{\partial t}+\left[\varphi, \psi-d_{e}^{2} \nabla_{\perp}^{2} \psi\right]-d_{\beta}[\psi, Z]+\frac{\partial \varphi}{\partial z}+d_{\beta} \frac{\partial Z}{\partial z}=0, \\
& \frac{\partial Z}{\partial t}+[\varphi, Z]-c_{\beta}[v, \psi]-d_{\beta}\left[\nabla_{\perp}^{2} \psi, \psi\right]-c_{\beta} \frac{\partial v}{\partial z}-d_{\beta} \frac{\partial \nabla_{\perp}^{2} \psi}{\partial z}=0, \\
& \frac{\partial \nabla_{\perp}^{2} \varphi}{\partial t}+\left[\varphi, \nabla_{\perp}^{2} \varphi\right]+\left[\nabla_{\perp}^{2} \psi, \psi\right]+\frac{\partial \nabla_{\perp}^{2} \psi}{\partial z}=0, \\
& \frac{\partial v}{\partial t}+[\varphi, v]-c_{\beta}[Z, \psi]-c_{\beta} \frac{\partial Z}{\partial z}=0,
\end{aligned}
$$

where the perpendicular gradient operator is defined by $\nabla_{\perp} f=(\partial f / \partial x) \hat{x}+(\partial f / \partial y) \hat{y}$. Note that, with the above assumptions, the 3D extension of the model is obtained from the $2 \mathrm{D}$ version, by mapping the expressions $[f, \psi]$, for a generic field $f$, into $[f, \psi]+\partial f / \partial z$. The model (22)-(25) can describe magnetic reconnection in three dimensions, in which case the Hamiltonian that describes the finite-dimensional system for magnetic field lines, $\psi(x, y, z, t)$, is in general non-integrable and can therefore lead to chaotic magnetic field lines (see, e.g. [21]). The $c_{\beta} \rightarrow 0, d_{\beta} \rightarrow \rho_{s}$ limit of (22)-(25) is also consistent with the $3 \mathrm{D}$ version of the two-field SPK model, which was investigated numerically in Refs. [7, 22, 14]. 
Analogously to the 2D version, this 3D model also admits a noncanonical Hamiltonian formulation. The latter can be conveniently expressed by making use of the normal fields of Eqs. (10)-(13) after replacing $\nabla$ with $\nabla_{\perp}$. Indeed, in these variables, the bracket for the 3D model has the relatively compact form

$$
\begin{aligned}
\{F, G\}=\int & d^{3} x\left(\zeta\left[F_{\zeta}, G_{\zeta}\right]+D\left(\left[F_{D}, G_{\zeta}\right]+\left[F_{\zeta}, G_{D}\right]\right)+T_{-}\left[F_{T_{-}}, G_{T_{-}}\right]\right. \\
& +T_{+}\left[F_{T_{+}}, G_{T_{+}}\right]+F_{\zeta} \frac{\partial}{\partial z} G_{D}+F_{D} \frac{\partial}{\partial z} G_{\zeta} \\
& \left.+\frac{d_{i}^{2}}{2 c_{\beta} d^{3} d_{e}} F_{T_{+}} \frac{\partial}{\partial z} G_{T_{+}}-\frac{d_{i}^{2}}{2 c_{\beta} d^{3} d_{e}} F_{T_{-}} \frac{\partial}{\partial z} G_{T_{-}}\right) .
\end{aligned}
$$

The Hamiltonian, on the other hand, still is given by the expression of Eq. (15), with the obvious extension of the integral and the fields to three dimensions, but $\nabla$ replaced by $\nabla_{\perp}$.

In terms of the normal field variables the model equations become

$$
\begin{aligned}
\frac{\partial D}{\partial t} & =-[\varphi, D]-\frac{\partial \varphi}{\partial z} \\
\frac{\partial \zeta}{\partial t} & =-[\varphi, \zeta]+d^{-2}[D, \psi]+\frac{1}{d} \frac{\partial}{\partial z}\left(D-d_{e} \mathcal{L}\left(\frac{d_{e}}{d^{2}} D+c_{\beta} d\left(T_{+}-T_{-}\right)\right)\right), \\
\frac{\partial T_{ \pm}}{\partial t} & =-\left[\varphi_{ \pm}, T_{ \pm}\right] \pm \frac{d_{i}^{2}}{2 c_{\beta} d^{3} d_{e}} \frac{\partial}{\partial z}\left(\frac{2 c_{\beta}^{2} d^{4}}{d_{i}^{2}} T_{ \pm}-\varphi \mp c_{\beta} d \mathcal{L}\left(\frac{d_{e}}{d^{2}} D+c_{\beta} d\left(T_{+}-T_{-}\right)\right)\right),
\end{aligned}
$$

from which it transpires that, in $3 \mathrm{D}$, the variables $D, \zeta, T_{+}$and $T_{-}$are no longer Lagrangian invariants.

Casimirs for the 3D bracket (26) are functionals $C$ such that

$$
\begin{aligned}
& \{F, C\}= \\
& \int d^{3} x\left[F_{\zeta}\left(\left[C_{\zeta}, \zeta\right]+\left[C_{D}, D\right]+\frac{\partial C_{D}}{\partial z}\right)+F_{D}\left(\left[C_{\zeta}, D\right]+\frac{\partial C_{\zeta}}{\partial z}\right)+\right. \\
& \left.F_{T_{+}}\left(\left[C_{T_{+}}, T_{+}\right]+\frac{d_{i}^{2}}{2 c_{\beta} d^{3} d_{e}} \frac{\partial C_{T_{+}}}{\partial z}\right)+F_{T_{-}}\left(\left[C_{T_{-}}, T_{-}\right]-\frac{d_{i}^{2}}{2 c_{\beta} d^{3} d_{e}} \frac{\partial C_{T_{-}}}{\partial z}\right)\right]=0
\end{aligned}
$$

for all functionals $F$. This implies Casimirs are solutions of the following system:

$$
\begin{aligned}
& {\left[C_{\zeta}, \zeta\right]+\left[C_{D}, D\right]+\frac{\partial C_{D}}{\partial z}=0} \\
& {\left[C_{\zeta}, D\right]+\frac{\partial C_{\zeta}}{\partial z}=0} \\
& {\left[C_{T_{+}}, T_{+}\right]+\frac{d_{i}^{2}}{2 c_{\beta} d^{3} d_{e}} \frac{\partial C_{T_{+}}}{\partial z}=0,} \\
& {\left[C_{T_{-}}, T_{-}\right]-\frac{d_{i}^{2}}{2 c_{\beta} d^{3} d_{e}} \frac{\partial C_{T_{-}}}{\partial z}=0 .}
\end{aligned}
$$

Equation (28), for instance, is of finite-dimensional Hamiltonian form, with $D$ playing the role of the Hamiltonian, $z$ playing the role of time, and the unknown $C_{\zeta}$ playing the 
role of the dynamical variable. Because we seek a solution for the case where $D$ depends on $z$, this is a 'time-dependent' Hamiltonian system. For general $D$ there is no solution of this equation besides the initial conditions, and these are not useful because they are not isolating, i.e. they do not define surfaces. The extension to three dimensions, therefore, eliminates the infinite families of Casimirs that were present in the 2D version.

Casimir solutions can, however, still be found and are given by

$$
C_{1}=\int d^{3} x D, \quad C_{2}=\int d^{3} x \zeta, \quad C_{3}=\int d^{3} x T_{+}, \quad C_{4}=\int d^{3} x T_{-} .
$$

The 3D model admits an additional constant of motion that is not a Casimir given by the functional

$$
h=\int d^{3} x\left(\zeta D+c_{\beta} d_{e} \frac{d^{3}}{d_{i}^{2}}\left(T_{+}^{2}-T_{-}^{2}\right)\right),
$$

which, in terms of the original fields, takes the form

$$
h=\int d^{3} x\left(\nabla_{\perp}^{2} \varphi \psi_{e}+\left(d_{i} \nabla_{\perp}^{2} \varphi+\frac{Z}{c_{\beta}}\right) v\right) .
$$

Note, if $Z=-d_{\beta} \nabla_{\perp}^{2} \varphi$, this quantity reduces to the generalized cross-helicity which is conserved in the 3D two-field SPK model [9].

\subsection{General extensions}

The 3D extension carried out for the FP model in Sec. 3.1 follows a path first travelled in Ref. [23], where an analogous extension was carried out for reduced magnetohydrodynamics (RMHD) [24]. The procedure for going from 2D to 3D can be formalized and applied to a large class of $2 \mathrm{D}$ models. Of particular interest for plasma physics is a class of $2 \mathrm{D}$ magnetofluid models, where the presence of a strong guide field is assumed. This class of models includes, besides the SPK, FP, and RMHD, the four-field model for tokamak dynamics of Hazeltine et al. [25], the model for iontemperature-gradient driven and drift Kelvin-Helmholtz modes of Waelbroeck et al. [26] or the more recent electromagnetic gyrofluid model of Waelbroeck et al. [19]. Indeed, for all such models, the extension to 3D amounts to extending a 2D Lie-Poisson bracket by including the dependence of the fields on the coordinate that was ignorable in the original 2D version. The general form of the extended Poisson bracket, for the class of models that includes all these models, has the form

$$
\begin{aligned}
\{F, G\} & =\{F, G\}_{\|}+\{F, G\}_{\perp} \\
& =\int d^{3} x A^{i j} F_{i} \partial G_{j}+\int d^{3} x W_{k}^{i j} \chi^{k}\left[F_{i}, G_{j}\right],
\end{aligned}
$$

where $\partial:=\partial / \partial z$, assuming that $z$ was the ignorable coordinate, and where $\chi^{1}, \cdots \chi^{n}$ indicate the field variables for an $n$-field model. Note $A^{i j}=A^{j i}$ and $W_{k}^{i j}=W_{k}^{j i}$, which are required for the bracket to be antisymmetric. The bracket is thus the sum of the bracket of the original 2D model, $\{F, G\}_{\perp}$, with a bracket that accounts for the 3D extension, $\{F, G\}_{\|}$. Not all antisymmetric bilinear operators of the form (34) are 
Poisson brackets, even if $\{F, G\}_{\perp}$ is a Poisson bracket. In fact, a Poisson bracket must also satisfy the Jacobi identity (see, e.g. $[27,8]$ )

$$
\{\{F, G\}, H\}+\{\{H, F\}, G\}+\{\{G, H\}, F\}=0,
$$

and this imposes restrictions on the elements of the matrix $A^{i j}$. In the following we derive these restrictions.

First, we obviously assume that the $W_{k}^{i j}$ have the commutation properties (see Ref. [28]) necessary for the Jacobi identity of $\{F, G\}_{\perp}$, i.e., we assume

$$
\left\{\{F, G\}_{\perp}, H\right\}_{\perp}+c y c \equiv 0,
$$

where $c y c$ indicates the addition of the two terms obtained by cyclic permutation of $F$, $G$, and $H$. By a theorem in Ref. [27], it follows for the form of $\{F, G\}_{\|}$given by (34) that

$$
\left\{\{F, G\}_{\|}, H\right\}_{\|}+c y c \equiv 0 .
$$

Therefore

$$
\begin{aligned}
\{\{F, G\}, H\}+c y c & =\left\{\{F, G\}_{\perp}, H\right\}_{\perp}+\left\{\{F, G\}_{\perp}, H\right\}_{\|} \\
& +\left\{\{F, G\}_{\|}, H\right\}_{\perp}+\left\{\{F, G\}_{\|}, H\right\}_{\|}+c y c .
\end{aligned}
$$

The first and last terms of (38) vanish by virtue of (36) and (37). The third term vanishes because

$$
\frac{\delta\{F, G\}_{\|}}{\delta \chi^{i}} \doteq 0
$$

where $\doteq$ means modulo second variation terms. Thus, it remains to show what the condition

$$
\{\{F, G\}, H\}+c y c=\left\{\{F, G\}_{\perp}, H\right\}_{\|}+c y c \equiv 0
$$

implies. Using

$$
\frac{\delta\{F, G\}_{\perp}}{\delta \chi^{k}} \doteq W_{k}^{i j}\left[F_{i}, G_{j}\right],
$$

we obtain

$$
\begin{aligned}
\left\{\{F, G\}_{\perp}, H\right\}_{\|}+c y c & \\
& \doteq \int d^{3} x A^{r s} W_{r}^{i j}\left(\left[F_{i}, G_{j}\right] \partial H_{s}+\left[G_{i}, H_{j}\right] \partial F_{s}+\left[H_{i}, F_{j}\right] \partial G_{s}\right) .
\end{aligned}
$$

Integrating the first term of (42) by parts gives

$$
\begin{gathered}
\left\{\{F, G\}_{\perp}, H\right\}_{\|}+c y c \doteq \int d^{3} x A^{r s} W_{r}^{i j}\left(-\left[\partial F_{i}, G_{j}\right] H_{s}-\left[F_{i}, \partial G_{j}\right] H_{s}\right. \\
\left.+\left[G_{i}, H_{j}\right] \partial F_{s}+\left[H_{i}, F_{j}\right] \partial G_{s}\right) .
\end{gathered}
$$

The first and third terms of (43) give

$$
\begin{aligned}
\int d^{3} x A^{r s} W_{r}^{i j} & \left(-\left[\partial F_{i}, G_{j}\right] H_{s}+\left[G_{i}, H_{j}\right] \partial F_{s}\right) \\
& =\int d^{3} x\left[G_{i}, H_{j}\right] \partial F_{s}\left(A^{r s} W_{r}^{i j}-A^{r j} W_{r}^{s i}\right),
\end{aligned}
$$


where the ' $f g h$-identity', $\int f[g, h]=-\int g[f, h]$, has been used and indices have been shifted. Similarly, the remaining two terms of (43) give

$$
\begin{aligned}
\int d^{3} x A^{r s} W_{r}^{i j}( & \left.-\left[F_{i}, \partial G_{j}\right] H_{s}+\left[H_{i}, F_{j}\right] \partial G_{s}\right) \\
& =\int d^{3} x\left[H_{i}, F_{j}\right] \partial G_{s}\left(A^{r s} W_{r}^{i j}-A^{r i} W_{r}^{j s}\right) .
\end{aligned}
$$

Because $\{\{F, G\}, H\}+c y c \equiv 0$ for all functionals $F, G, H$, we obtain from (44) and (45) the following compatibility conditions on $A^{i j}$, needed for the Jacobi identity:

$$
A^{r s} W_{r}^{i j}=A^{r j} W_{r}^{s i}=A^{r i} W_{r}^{j s} .
$$

In the specific case of the FP model, if we choose

$$
\chi^{1}=D, \quad \chi^{2}=\zeta, \quad \chi^{3}=T_{+}, \quad \chi^{4}=T_{-},
$$

and then obtain

$$
A=\left(\begin{array}{cccc}
0 & 1 & 0 & 0 \\
1 & 0 & 0 & 0 \\
0 & 0 & a & 0 \\
0 & 0 & 0 & -a
\end{array}\right)
$$

with $a=d_{i}^{2} /\left(2 c_{\beta} d^{3} d_{e}\right)$ and the only non-vanishing elements of $W_{k}^{i j}$ are

$$
W_{2}^{22}=W_{1}^{12}=W_{1}^{21}=W_{3}^{33}=W_{4}^{44}=1
$$

Therefore, the condition (46) is satisfied and (26) is indeed a Poisson bracket.

\subsection{Addition of external field perturbations}

The 3D four-field model (22)-(25) can be extended to include the presence of external perturbations in such a way that the resulting extended model can be shown to still admit a Hamiltonian formulation. Indeed, we can obtain a system driven by an external perturbation by adding to the Hamiltonian a term of the form

$$
H_{e x t}=\int d^{3} x \chi^{i} D_{i j} \chi_{e x t}^{j}(x, y, z, t),
$$

where $\chi_{\text {ext }}^{j}$ indicates the external fields and $\left(D_{i j}\right)$ is a constant matrix. Keeping the same Poisson bracket, the model equations for the externally perturbed system are then given by

$$
\frac{\partial \chi^{i}}{\partial t}=\left\{\chi^{i}, H+H_{e x t}\right\}
$$

It is important to note that with this form for the drive, the Casimir invariants remain constants of motion, because it still follows that

$$
\left\{C, H+H_{e x t}\right\}=0,
$$

i.e. the drive allows one to reach only dynamically accessible states. Energy is added to (or subtracted from) the system according to the following:

$$
\frac{d H}{d t}=\left\{H, H_{e x t}\right\},
$$


and

$$
\Delta H_{e x t}=\int d t \frac{d H_{e x t}}{d t}=\int d t\left(\frac{\partial H_{e x t}}{\partial t}+\left\{H_{e x t}, H\right\}\right) .
$$

As an example, we present an extended version of the 3D four-field model that takes into account the effect of external magnetic poloidal perturbations and that still possesses the Hamiltonian structure. This can be obtained by considering the Hamiltonian

$$
\begin{aligned}
\bar{H} & =H+H_{e x t} \\
& =\frac{1}{2} \int_{\mathcal{D}} d^{3} x\left(d_{e}^{2} J^{2}+|\nabla \varphi|^{2}+v^{2}+|\nabla \psi|^{2}+Z^{2}+\psi_{\text {ext }} \psi_{e}\right),
\end{aligned}
$$

where $\psi_{\text {ext }}$ is the external magnetic perturbation. This leads to the following model:

$$
\begin{aligned}
& \frac{\partial\left(\psi-d_{e}^{2} \nabla_{\perp}^{2} \psi\right)}{\partial t}+\left[\varphi, \psi-d_{e}^{2} \nabla_{\perp}^{2} \psi\right]-d_{\beta}[\psi, Z]+\frac{\partial \varphi}{\partial z}+d_{\beta} \frac{\partial Z}{\partial z}= \\
& -d_{\beta} d_{e}^{2}\left[\psi_{\text {ext }}, Z\right] \\
& \frac{\partial Z}{\partial t}+[\varphi, Z]-c_{\beta}[v, \psi]-d_{\beta}\left[\nabla_{\perp}^{2} \psi, \psi\right]-c_{\beta} \frac{\partial v}{\partial z}-d_{\beta} \frac{\partial \nabla_{\perp}^{2} \psi}{\partial z}= \\
& -d_{\beta}\left[\psi_{\text {ext }}, \psi\right]+d_{\beta} d_{e}^{2}\left[\psi_{\text {ext }}, \nabla_{\perp}^{2} \psi\right]+c_{\beta} d_{e}^{2}\left[\psi_{\text {ext }}, v\right]-d_{\beta} \frac{\partial \psi_{\text {ext }}}{\partial z}, \\
& \frac{\partial \nabla_{\perp}^{2} \varphi}{\partial t}+\left[\varphi, \nabla_{\perp}^{2} \varphi\right]+\left[\nabla_{\perp}^{2} \psi, \psi\right]+\frac{\partial \nabla_{\perp}^{2} \psi}{\partial z}= \\
& {\left[\psi_{e x t}, \psi\right]-d_{e}^{2}\left[\psi_{e x t}, \nabla_{\perp}^{2} \psi\right]+\frac{\partial \psi_{e x t}}{\partial z}} \\
& \frac{\partial v}{\partial t}+[\varphi, v]-c_{\beta}[Z, \psi]-c_{\beta} \frac{\partial Z}{\partial z}= \\
& c_{\beta} d_{e}^{2}\left[\psi_{\text {ext }}, Z\right] \text {. }
\end{aligned}
$$

Evidently, there are two kinds of drive here, additive and multiplicative, and so there are various possibilities. For example, one can temporally drive a $2 \mathrm{D}$ theory additively by supposing

$$
\psi_{\text {ext }}=z \Psi(x, y, t)
$$

with $\Psi$ some prescribed 2D perturbing magnetic flux.

We point out that such extension to externally driven Hamiltonian models, obtained by using (50), can be applied to all Hamiltonian plasma models Poisson brackets, and in particular those of the form of (34).

\section{Conclusions}

In this paper, different aspects of the nonlinear dynamics and modelling of magnetic reconnection in collisionless plasmas have been considered. In Sec. 2 we addressed the question of seeing whether the vorticity phase mixing process and the layer formations, that were observed in a simpler model valid at very low $\beta$, persist at higher $\beta$ values, which can be accessed by the FP model. Numerical simulations of the FP model showed 
that at very low values of $\beta$, and when $d_{\beta} \approx \rho_{s}$, also parallel magnetic perturbations undergo a phase-mixing process in which energy is transferred to small scales. On the other hand, we also showed that this process tends to disappear when higher $\beta$ regimes are entered. This behavior was naturally explained in terms of the growth of the normal field $\zeta$, which also measures the deviation from proportionality between the parallel magnetic perturbation and the vorticity.

In Sec. 3, we presented an extension of the FP model to three-dimensions. We proved that the 3D extension still possesses a Hamiltonian structure, but that the number of Casimir invariants was dramatically reduced. Next, a condition was derived for determining 3D extensions for a large class of 2D plasma models, which preserve the Hamiltonian structure. Finally, we showed how such 3D Hamiltonian models can also account for externally applied fields.

Numerical simulations of such extended models in the presence of external fields, and the investigation of the resulting nonlinear dynamics, will be a subject of future work.

\section{References}

[1] D. Biskamp. Magnetic Reconnection in Plasmas. Cambridge University Press, Cambridge, 2000.

[2] E. R. Priest and T. G. Forbes. Magnetic Reconnection. Cambridge University Press, Cambridge, 2000.

[3] T. J. Schep, F. Pegoraro, and B. N. Kuvshinov. Generalized two-fluid theory of nonlinear magnetic structures. Phys. Plasmas, 1:2843-2851, 1994.

[4] E. Cafaro, D. Grasso, F. Pegoraro, F. Porcelli, and A. Saluzzi. Invariants and geometric structures in nonlinear Hamiltonian magnetic reconnection. Phys. Rev. Lett., 80:4430-4433, 1998.

[5] D. Grasso, F. Califano, F. Pegoraro, and F. Porcelli. Phase mixing and saturation in Hamiltonian reconnection. Phys. Rev. Lett., 86:5051-5054, 2001.

[6] F. Porcelli, D. Borgogno, F. Califano, D. Grasso, M. Ottaviani, and F. Pegoraro. Recent advances in collisionless magnetic reconnection. Plasma Phys. Control. Fusion, 44:389-406, 2002.

[7] D. Borgogno, D. Grasso, F. Califano, D. Farina, F. Pegoraro, and F. Porcelli. Aspects of threedimensional magnetic reconnection. Phys. Plasmas, 12:032309, 2005.

[8] P. J. Morrison. Hamiltonian description of the ideal fluid. Rev. Mod. Phys., 70:467-521, 1998.

[9] B.N. Kuvshinov, F. Pegoraro, and T.J. Schep. Hamiltonian formulation of low-frequency, nonlinear plasma dynamics. Phys. Lett. A, 191 A:296, 1994.

[10] R. Fitzpatrick and F. Porcelli. Collisionless magnetic reconnection with arbitrary guide field. Phys. Plasmas, 11:4713-4718, 2004. Erratum: 14, 049902 (2007).

[11] E. Tassi, P.J. Morrison, F.L. Waelbroeck, and D. Grasso. Hamiltonian formulation and analysis of a collisionless fluid reconnection model. Plasma Phys. Control. Fusion, 50:085014, 2008.

[12] E. Tassi, D. Grasso, F. Pegoraro, and P.J. Morrison. Stability and nonlinear dynamics aspects of a model for collisionless magnetic reconnection. Journal of Plasma and Fusion Research Series, 2008. accepted for publication.

[13] E. Tassi, D. Grasso, and F. Pegoraro. Nonlinear processes in Hamiltonian reconnection. Commun. Nonlinear Sci. Numer. Simulat., $\underline{15}: \underline{2}, \underline{2010}$.

[14] D. Grasso, D. Borgogno, F. Pegoraro, and E. Tassi. Coupling between reconnection and KelvinHelmholtz instabilities in collisionless plasmas. Nonlin. Processes Geophys., 16:241-249, 2009.

[15] D. Reiser and B. Scott. Electromagnetic fluid drift turbulence in static ergodic magnetic fields. Phys. Plasmas, 12:122308, 2005. 
[16] D. Reiser. Impact of large island perturbations on turbulent blob transport in tokamaks. Phys. Plasmas, 14:082314, 2007.

[17] D. Reiser and D. Chandra. Plasma currents induced by resonant magnetic field perturbations in tokamaks. Phys. Plasmas, 16:042317, 2009.

[18] E. Tassi, P. J. Morrison, and D. Grasso. Hamiltonian structure of a collisionless reconnection model valid for high and low $\beta$ plasmas. In G. Bertin, R. Pozzoli, M. Rome, and K. R. Sreenivasan, editors, Collective phenomena in macroscopic systems, pages 197-206. World Scientific, Singapore, 2007.

[19] F.L. Waelbroeck, R.D. Hazeltine, and P.J. Morrison. A Hamiltonian electromagnetic gyrofluid model. Phys. Plasmas, 16:032109, 2009.

[20] D. Del Sarto, F. Califano, and F. Pegoraro. Secondary instabilities and vortex formation in collisionless-fluid magnetic reconnection. Phys. Rev. Lett., 91(23):235001, 2003.

[21] P. J. Morrison. Magnetic field lines, Hamiltonian dynamics, and nontwist systems. Phys. Plasmas, 7:2279-2289, 2000.

[22] D. Grasso, D. Borgogno, and F. Pegoraro. Secondary instabilities in two and three-dimensional magnetic reconnection in fusion relevant plasmas. Phys. Plasmas, 14:055703, 2007.

[23] P. J. Morrison and R. D. Hazeltine. Hamiltonian formulation of reduced magnetohydrodynamics. Phys. Fluids, 27:886-897, 1984.

[24] R. Strauss. Nonlinear three-dimensional magnetohydrodynamics of noncircular tokamaks. Phys. Fluids, 19:134, 1976.

[25] R. D. Hazeltine, C. T. Hsu, and P. J. Morrison. Hamiltonian four-field model for nonlinear tokamak dynamics. Phys. Fluids, 30:3204-3211, 1987.

[26] F.L. Waelbroeck, P. J. Morrison, and W. Horton. Hamiltonian formulation and coherent structures in electrostatic turbulence. Plasma Phys. Contr. Fusion, 46:1331-1350, 2004.

[27] P. J. Morrison. Poisson brackets for fluids and plasmas. In M. Tabor and Y. Treve, editors, Mathematical Methods in Hydrodynamics and Integrability in Dynamical Systems, volume 88 of American Institute of Physics Conference Proceedings, pages 13-45. American Institute of Physics, 1982.

[28] J. L. Thiffeault and P. J. Morrison. Classification and Casimir invariants of Lie-Poisson brackets. Physica D, 136:205-244, 2000. 